PROCEEDINGS OF THE

AMERICAN MATHEMATICAL SOCIETY

Volume 136, Number 9, September 2008, Pages 3327-3330

S 0002-9939(08)09275-7

Article electronically published on April 29, 2008

\title{
PARAMETER-FREE UNIFORMISATION
}

\author{
SY-DAVID FRIEDMAN
}

(Communicated by Julia Knight)

\begin{abstract}
This article was motivated by two questions, one in the theory of infinite-time Turing machines and the other a fine-structural question posed by Wayne Richter. The answers to both turn on a question of parameter-free uniformisation, which I prove for all locally countable, limit initial segments of $L$.
\end{abstract}

Let $\alpha$ be a limit ordinal. We say that the partial function $f$ on $L_{\alpha}$ uniformises the binary relation $R(x, y)$ on $L_{\alpha}$ iff for all $p \in L_{\alpha}: p \in \operatorname{Dom}(f)$ iff $\exists y R(p, y)$ iff $R(p, f(p))$. Then $L_{\alpha}$ satisfies $\Sigma_{n}$ uniformisation iff every binary relation $R(x, y)$ which is $\Sigma_{n}$ definable over $L_{\alpha}$ with parameters can be uniformised by a function $f$ which is $\Sigma_{n}$ definable over $L_{\alpha}$ with parameters. A stronger statement is parameterfree $\Sigma_{n}$ uniformisation, which asserts the same for relations and functions without parameters. Jensen proved that each $L_{\alpha}$ satisfies $\Sigma_{n}$ uniformisation (see 3]). There are examples of $L_{\alpha}$ 's which do not satisfy parameter-free $\Sigma_{2}$ uniformisation (see [1]). Every $L_{\alpha}$ satisfies parameter-free $\Sigma_{1}$ uniformisation.

We say that $\beta$ is $\Sigma_{1}$ stable in $\alpha$ iff $\beta$ is less than $\alpha$ and $L_{\beta}$ is $\Sigma_{1}$ elementary in $L_{\alpha}$. We say that $\beta$ is above $x$ iff $x$ belongs to $L_{\beta}$.

Theorem 1. Suppose that $\alpha$ is a limit ordinal and $L_{\alpha}$ is locally countable (i.e., $L_{\alpha} \vDash$ every set is countable). Then $L_{\alpha}$ satisfies parameter-free $\Sigma_{n}$ uniformisation.

Proof. By induction on $n$. We may assume that $n$ is greater than 1 . For the sake of clarity, we assume that $n$ equals 2 ; the proof below readily generalises to arbitrary $n$, given the result for $n-1$.

We first discuss $\Sigma_{1}$ Skolem functions. Let $U$ be the set of pairs $(\langle n, x\rangle, y)$ in $L_{\alpha}$ such that $\varphi_{n}(x, y)$ holds in $L_{\alpha}$, where $\varphi_{n}$ is the $n$-th $\Sigma_{1}$ formula. Then $U$ is parameter-free $\Sigma_{1}$ and can be uniformised by a parameter-free $\Sigma_{1}$ function $h_{1}$. Thus the domain of $h_{1}$ consists of those pairs $\langle n, x\rangle$ such that $\varphi_{n}(x, y)$ holds for some $y$, and for such a pair, $\varphi_{n}\left(x, h_{1}(\langle n, x\rangle)\right)$ holds $\left(h_{1}\right.$ is a $\Sigma_{1}$ Skolem function for $\left.L_{\alpha}\right)$. It is easy to verify that for any $A \subseteq L_{\alpha}$, the range of $h_{1}$ on $\omega \times[A]^{<\omega}$ is the smallest $\Sigma_{1}$ elementary submodel of $L_{\alpha}$ containing $A$ as a subset, the $\Sigma_{1}$ Skolem hull of $A$. For any $A \subseteq L_{\alpha}$ and $x \in L_{\alpha}$, the $\Sigma_{1}$ Skolem hull of $A \cup\{x\}$ is equal to the set of all $h_{1}(n,\langle x, a\rangle), n \in \omega$ and $a \in[A]^{<\omega}$.

Received by the editors February 28, 2007, and, in revised form, July 17, 2007.

2000 Mathematics Subject Classification. Primary 03E35, 03E45, 03E55.

Key words and phrases. Descriptive set theory, large cardinals, innermodels.

The author was supported by Grants P 16790-NO4 and P 19375-N18 of the Austrian Science Fund (FWF).

(C)2008 American Mathematical Society Reverts to public domain 28 years from publication 
As $L_{\alpha}$ is locally countable, the $\Sigma_{1}$ Skolem hull of $A$ in $L_{\alpha}$ is transitive for any $A \subseteq L_{\alpha}$ : If this hull contains $x$, then it also contains an injection of $x$ into $\omega$, all elements of $\omega$ and therefore all elements of $x$. It follows that the $\Sigma_{1}$ Skolem hull of $\{\beta, x\}$ equals the $\Sigma_{1}$ Skolem hull of $\beta+1 \cup\{x\}$ for any $\beta<\alpha$ and $x \in L_{\alpha}$.

Lemma 2. Suppose that $y$ belongs to $L_{\alpha}$. Then for some $n \in \omega, y$ equals $h_{1}(n, \beta)$ where $\beta$ is either 0 or $\Sigma_{1}$ stable in $\alpha$. More generally, for any $x \in L_{\alpha}$, there exists $n \in \omega$ such that $y$ equals $h_{1}(n,\langle\beta, x\rangle)$ where $\beta$ is either 0 or both $\Sigma_{1}$ stable in $\alpha$ and above $x$.

Proof. Given $y$, let $\beta$ be least so that $y$ belongs to the $\Sigma_{1}$ Skolem hull of $\beta+1$ in $L_{\alpha}$ (= the $\Sigma_{1}$ Skolem hull of $\{\beta\}$ in $L_{\alpha}$ ). Then $\beta$ is either 0 or $\Sigma_{1}$ stable in $\alpha$, as otherwise $\beta$ belongs to the $\Sigma_{1}$ Skolem hull of $\beta^{\prime}+1$ for some $\beta^{\prime}<\beta$ and hence so does $y$, contradicting the choice of $\beta$. Also, $y$ is of the form $h_{1}(n, \beta)$ for some $n \in \omega$. More generally, given both $y$ and $x$ in $L_{\alpha}$, let $\beta$ be least so that $y$ belongs to the $\Sigma_{1}$ Skolem hull of $\beta+1 \cup\{x\}$ in $L_{\alpha}$ (= the $\Sigma_{1}$ Skolem hull of $\{\beta, x\}$ in $L_{\alpha}$ ). As the $\Sigma_{1}$ Skolem hull of $1 \cup\{x\}$ is transitive, it follows that $\beta$ is either 0 or $\Sigma_{1}$ stable and above $x$. Also, $y$ is equal to $h_{1}(n,\langle\beta, x\rangle)$ for some $n$.

Now let $\psi_{n}(x, y)$ be the $n$-th $\Pi_{1}$ formula with free variables $x, y$ and define $f(n, x)=(m, \beta)$ iff the following hold in $\left(L_{\alpha}, \in\right)$ :

(i) Either $\beta$ is 0 or both $\Sigma_{1}$ stable and above $x$.

(ii) $h_{1}(\langle m,\langle\beta, x\rangle\rangle)=y$ is defined.

(iii) $\psi_{n}\left(x, h_{1}(\langle m,\langle\beta, x\rangle\rangle)\right)$ holds.

(iv) $\psi_{n}\left(x, y^{\prime}\right)$ fails for any $y^{\prime}$ of the form $h_{1}\left(\left\langle m^{\prime},\left\langle\beta^{\prime}, x\right\rangle\right\rangle\right), \beta^{\prime}<\beta, m^{\prime}<\omega$.

(v) $m^{\prime}<m \rightarrow h_{1}\left(\left\langle m^{\prime},\langle\beta, x\rangle\right\rangle\right)$ is undefined or $\psi_{n}\left(h_{1}\left(\left\langle m^{\prime},\langle\beta, x\rangle\right\rangle\right)\right)$ fails.

Clauses (i)-(iii) are easily seen to be equivalent to a Boolean combination of $\Sigma_{1}$ formulas, using the fact that $\Sigma_{1}$ stability is a $\Pi_{1}$ notion. Clause (iv) is vacuous if $\beta$ is 0 and otherwise, by the fact $\beta$ is $\Sigma_{1}$ stable and above $x$, holds in $\left(L_{\alpha}, \in\right)$ iff it holds in $\left(L_{\beta}, \in\right)$; it follows that clause (iv) is $\Sigma_{1}$. Also, clause (v) is a Boolean combination of $\Sigma_{1}$ formulas preceded by a bounded number quantifier; it follows that this clause is $\Sigma_{2}$. So $f$ is a partial function from $L_{\alpha}$ to $L_{\alpha}$ which is parameterfree $\Sigma_{2}$.

If $f(n, x)$ is defined, then $\psi_{n}(x, y)$ holds for some $y$, as we may take $y$ to be $h_{1}(\langle m,\langle\beta, x\rangle\rangle)$, where $f(n, x)=(m, \beta)$. Conversely, if $\psi_{n}(x, y)$ holds for some $y$, then by the lemma, $y$ is of the form $h_{1}(\langle m,\langle\beta, x\rangle\rangle)$ for some $\beta$ which is either 0 or both $\Sigma_{1}$ stable and above $x$. By minimising the pair $(\beta, m)$, we see that $f(n, x)$ is defined. Thus $f(n, x)$ is defined iff for some $y, \psi_{n}(x, y)$ holds, in which case $\psi_{n}\left(x, h_{1}(\langle m,\langle\beta, x\rangle\rangle)\right)$ holds, where $f(n, x)=(m, \beta)$. For each $(n, x)$ define $f^{\prime}(n, x)$ to be $h_{1}(\langle m,\langle\beta, x\rangle\rangle)$, where $f(n, x)=(m, \beta)$. Then $f^{\prime}$ is parameter-free $\Sigma_{2}$ with the same domain as $f$, and $f^{\prime}(n, x)$ is defined iff for some $y, \psi_{n}(x, y)$ holds, in which case $\psi_{n}\left(x, f^{\prime}(n, x)\right)$ holds.

Now suppose that $R(x, y)$ is a binary relation on $L_{\alpha}$ which is $\Sigma_{2}$ definable over $L_{\alpha}$ without parameters. Let $R(x, y)$ be defined over $L_{\alpha}$ by the $\Sigma_{2}$ formula $\exists z \psi(x, y, z)$, where $\psi$ is $\Pi_{1}$. Now let $R^{\prime}$ consist of all pairs $(x,\langle y, z\rangle)$ such that $\psi(x, y, z)$ holds in $L_{\alpha}$; then $R^{\prime}$ is $\Pi_{1}$ definable over $L_{\alpha}$. Choose $n$ so that $R^{\prime}$ is defined over $L_{\alpha}$ by the $\Pi_{1}$ formula $\psi_{n}$. Then $f^{\prime}(n, x)$ is defined iff $(x,\langle y, z\rangle)$ belongs to $R^{\prime}$ for some $y, z$, in which case $\left(x, f^{\prime}(n, x)\right)$ belongs to $R^{\prime}$. Let $g$ be the partial function defined by

$$
g(x)=y \text { iff for some } z, f^{\prime}(n, x) \text { is the pair }\langle y, z\rangle .
$$


Then $g$ is parameter-free $\Sigma_{2}$ and $x$ is in the domain of $g$ iff $f^{\prime}(n, x)$ is a pair $\langle y, z\rangle$ where $(x,\langle y, z\rangle)$ belongs to $R^{\prime}$ iff $(x,\langle g(x), z\rangle)$ belongs to $R^{\prime}$ for some $z$ iff $R(x, g(x))$ holds. So $g$ uniformises $R$, as desired.

Remark. The hypothesis of local countability can be weakened slightly. All that was needed for the proof was the transitivity of $\Sigma_{1}$ Skolem hulls, which holds iff $L_{\alpha}$ is locally countable or is the $\Sigma_{1}$ Skolem hull of $\left\{\omega_{1}^{L_{\alpha}}\right\}$ in itself.

\section{Applications}

Wayne Richter asked the following question: Suppose that $\alpha$ is least so that for some $\kappa<\alpha, L_{\kappa}$ and $L_{\alpha}$ have the same $\Sigma_{n}$ theory. Then is $L_{\kappa}$ a $\Sigma_{n}$ elementary submodel of $L_{\alpha}$ ?

The answer is Yes, as was independently verified by Leo Harrington and myself in 2003. Here I give a new proof based on Theorem 1 .

Corollary 3. Suppose that $\alpha$ is least so that for some $\kappa<\alpha, L_{\kappa}$ and $L_{\alpha}$ have the same $\Sigma_{n}$ theory. Then $L_{\kappa}$ is a $\Sigma_{n}$ elementary submodel of $L_{\alpha}$.

Proof. Note that $\alpha$ is a limit ordinal; otherwise both $\kappa$ and $\alpha$ are successor ordinals and $L_{\kappa-1}, L_{\alpha-1}$ have the same theory, contradicting the leastness of $\alpha$. Also, $L_{\alpha}$ is locally countable, as if $\beta=\left(\omega_{1}\right)^{L_{\alpha}}$ exists, there are $\kappa<\beta$ such that $L_{\kappa}$ is $\Sigma_{n}$ is elementary in $L_{\beta}$, again contradicting the leastness of $\alpha$. So $\alpha$ satisfies the hypotheses of Theorem [1. By that theorem, $L_{\alpha}$ has a parameter-free $\Sigma_{n}$ Skolem function, i.e., there exists a parameter-free $\Sigma_{n}$ partial function $h_{n}$ from $\omega \times L_{\alpha}$ into $L_{\alpha}$ such that for any $A \subseteq L_{\alpha}$, the set of $h_{n}(m, a)$ for $m \in \omega$ and $a \in[A]^{<\omega}$ is the smallest $\Sigma_{n}$ elementary submodel of $L_{\alpha}$ containing $A$ as a subset, the $\Sigma_{n}$ Skolem hull of $A$. Let $H$ be the $\Sigma_{n}$ Skolem hull of $\omega$ in $L_{\alpha}$. Then $H$ is transitive and is $\Sigma_{n}$ elementary in $L_{\alpha}$. Therefore $H$ is an initial segment of $L$ with the same $\Sigma_{n}$ theory as $L_{\alpha}$, and it therefore must be either $L_{\kappa}$ or $L_{\alpha}$. Note that each element of $H$ is a $\Sigma_{n}$ singleton in $L_{\alpha}$ (i.e., the unique solution to a $\Sigma_{n}$ formula in $L_{\alpha}$ ) as each element of $H$ is of the form $h_{n}(m, a)$ where $(m, a) \in \omega \times \omega^{<\omega}$ and $h_{n}$ is parameter-free $\Sigma_{n}$. If $H$ equals $L_{\alpha}$, then $\kappa$ belongs to $H$ and so we can choose a $\Sigma_{n}$ formula $\psi(x)$ whose unique solution in $L_{\alpha}$ is $\kappa$. But then the $\Pi_{n}$ theory of $L_{\alpha}$ is $\Sigma_{n}$ definable over $L_{\alpha}$ : $\varphi$ belongs to the $\Pi_{n}$ theory of $L_{\alpha}$ iff for some $\beta, \varphi$ is true in $L_{\beta}$ and $\psi(\beta)$ holds. The latter is a contradiction. So $H$ equals $L_{\kappa}$ and therefore $L_{\kappa}$ is $\Sigma_{n}$ elementary in $L_{\alpha}$.

Our second application is to infinite-time Turing machines. In [2], the Theory Machine is defined, which is a Turing machine which runs through the transfinite and at stage $\omega^{2} \times(\alpha+1)$ writes down the $\Sigma_{2}$ theory of $J_{\alpha}$. The machine can continue doing this until it reaches a limit stage $\Sigma$ such that there is no parameter-free $\Sigma_{2}$ definable partial function from $\omega$ onto $L_{\Sigma}$. This ordinal $\Sigma$ is described as follows.

Corollary 4. Let $\Sigma$ be least such that for some $\kappa<\Sigma, L_{\kappa}$ is $\Sigma_{2}$ elementary in $L_{\Sigma}$ (by the previous corollary, this is the least $\Sigma$ such that for some $\kappa<\Sigma, L_{\kappa}$ and $L_{\Sigma}$ have the same $\Sigma_{2}$ theory). Then $\Sigma$ is the least limit ordinal so that there is no parameter-free $\Sigma_{2}$ definable partial function from $\omega$ onto $L_{\Sigma}$, and therefore the Theory Machine can write down $\Sigma_{2}$ theories for $\Sigma$ steps.

Proof. Note that $L_{\alpha}$ is locally countable for $\alpha \leq \Sigma$, where if $\beta=\left(\omega_{1}\right)^{L_{\alpha}}$ exists, then there are $\kappa<\beta$ such that $L_{\kappa}$ is $\Sigma_{2}$ elementary in $L_{\beta}$, contradicting the definition of $\Sigma$. So by Theorem 1, $L_{\alpha}$ has a parameter-free $\Sigma_{2}$ Skolem function for each limit 
$\alpha \leq \Sigma$. For such $\alpha$ let $H$ be the $\Sigma_{2}$ Skolem hull of $\omega$ in $L_{\alpha}$. Then $H$ is a limit initial segment of $L$ and therefore if $\alpha$ is less than $\Sigma, H$ equals $L_{\alpha}$. It follows that for limit $\alpha<\Sigma$, there is a parameter-free $\Sigma_{2}$ definable partial function from $\omega$ onto $L_{\alpha}$. There is no such function for $\Sigma$, as the range of any such function must be contained in $L_{\kappa}$, where $L_{\kappa}$ is $\Sigma_{2}$ elementary in $L_{\Sigma}$ and $\kappa$ is less than $\Sigma$.

Remarks. (i) The previous corollary does not need the full power of Theorem 1 . It only needs that for limit $\alpha \leq \Sigma$ there is a parameter-free $\Sigma_{2}$ definable partial function from $\omega$ onto the least $\Sigma_{2}$ elementary submodel of $L_{\alpha}$. (For any limit $\alpha$, there is a least $\Sigma_{2}$ elementary submodel of $L_{\alpha}$, namely the set of $L$-least elements of nonempty $\Sigma_{2}$ definable subsets of $L_{\alpha}$.) This weaker property holds whenever $L_{\alpha}$ is the $\Sigma_{1}$ Skolem hull of $\{\beta\}$, where $\beta$ is the largest $\Sigma_{1}$ stable in $L_{\alpha}$ (should this exist).

(ii) Regarding the $\kappa$ and $\Sigma$ of the previous corollary: $\kappa$ must be the largest $\Sigma_{1}$ stable in $\Sigma$, as any larger $\Sigma_{1}$ stable would yield the same $\Sigma_{2}$ theory as $\Sigma$, in contradiction to the leastness of $\Sigma$. Also, $\Sigma$ is not $\Sigma_{1}$ admissible, as otherwise there would be an $\bar{\Sigma}<\Sigma$ such that $L_{\bar{\Sigma}}$ satisfies all $\Pi_{2}$ sentences true in $L_{\Sigma}$ (since this set of sentences belongs to $L_{\Sigma}$ ); but then $L_{\bar{\Sigma}}, L_{\Sigma}$ have the same $\Sigma_{2}$ theory, again contradicting the leastness of $\Sigma$. So in fact there is a bijection between $\omega$ and $\Sigma$ which is $\Sigma_{1}$ definable over $L_{\Sigma}$ with parameter $\kappa$.

Question. For exactly which limit $\alpha$ does there exist a parameter-free $\Sigma_{n}$ Skolem function for $L_{\alpha}$ ?

\section{REFERENCES}

[1] Friedman, S. Fine structure and class forcing, de Gruyter, 2000. MR 1780138 (2001g:03001)

[2] Friedman, S. and Welch, P.D., Two observations regarding infinite-time Turing machines, preprint.

[3] Jensen, R. The fine structure of the constructible hierarchy, Annals of Mathematical Logic 4, pp. 229-308, 1972. MR0309729 (46:8834)

[4] Welch, P.D. Eventually infinite time Turing machine degrees: infinite time decidable reals, Journal of Symbolic Logic 65, no. 3, pp. 1193-1203, 2000. MR 1791371 (2002b:03093)

Kurt Gödel Research Center, Universität Wien, Währingerstrasse 25, A-1010 Wien, AUstria

E-mail address: sdf@logic.univie.ac.at 